\title{
Correction to: Survival of Xanthomonas campestris pv. campestris associated with soil and cauliflower crop debris under Brazilian conditions
}

\author{
T. A. F. Silva Júnior • J. C. Silva • R. M. Gonçalves • J.
}

M. Soman • J. R. S. Passos • A. C. Maringoni

Published online: 19 May 2020

(C) Koninklijke Nederlandse Planteziektenkundige Vereniging 2020

\section{Correction to: Eur J Plant Pathol https://doi.org/10.1007/s10658-019-01888-8}

Author family name to be read as Silva Júnior.

The online version of the original article can be found at https://doi.org/10.1007/s10658-019-01888-8

T. A. F. S. Júnior $(\bowtie) \cdot J$ J. C. Silva · R. M. Gonçalves • J. M. Soman - A. C. Maringoni

Departamento de Proteção Vegetal, Faculdade de Ciências Agronômicas (FCA), Universidade Estadual Paulista "Júlio de Mesquita Filho" (UNESP), Avenida Universitária, 3780,

Botucatu, SP 18610-034, Brazil

e-mail: adeusilvajr@gmail.com

J. R. S. Passos

Departamento de Bioestatística, Instituto de Biociências, UNESP,

P.O. Box 510, Botucatu, São Paulo 18618-970, Brazil 